\title{
Antimicrobial Activity of Colloidal Selenium Nanoparticles in Chitosan Solution against Streptococcus mutans, Lactobacillus acidophilus, and Candida albicans
}

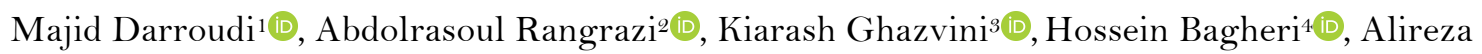 \\ Boruziniat ${ }^{2}$ (1)
}

\begin{abstract}
${ }^{1}$ Nuclear Medicine Research Center, Mashhad University of Medical Sciences, Mashhad, Iran.
${ }^{2}$ Dental Research Center, Mashhad University of Medical Sciences, Mashhad, Iran.

${ }^{3}$ Antimicrobial Resistance Research Center, Mashhad University of Medical Sciences, Mashhad, Iran.

${ }^{4}$ Dental Materials Research Center, Mashhad University of Medical Sciences, Mashhad, Iran.
\end{abstract}

Correspondence: Abdolrasoul Rangrazi, Assistant Professor, Dental Research Center, Mashhad University of Medical Sciences, Mashhad, Iran. E-mail: rangrazi.r@gmail.com

Academic Editor: Catarina Ribeiro Barros de Alencar

Received: 05 June 2020 / Review: 26 October 2020 / Accepted: 28 December 2020

How to cite: Darroudi M, Rangrazi A, Ghazvini K, Bagheri H, Boruziniat A. Antimicrobial activity of colloidal selenium nanoparticles in chitosan solution against Streptococcus mutans, Lactobacillus acidophilus, and Candida albicans. Pesqui Bras Odontopediatria Clín Integr. 202 1; 21 :e0121. https://doi.org/10.1590/pboci.202 1.069

\begin{abstract}
Objective: To investigate the antimicrobial activity of colloidal selenium nanoparticles in chitosan solution (Cts-Se-NPs) against Streptococcus mutans, Lactobacillus acidophilus, and Candida albicans. Material and Methods: Cts-Se-NPs solution was prepared using a simple chemical reduction method. The MIC and MBC against $S$. mutans, L. acidophilus, and C. albicans were determined using the broth dilution assay. Results: The Cts-Se-NPs had remarkable antimicrobial activity against $S$. mutans, L. acidophilus, and $C$. albicans. The MIC values of the Cts-Se-NPs were lowest for $S$. mutans $(0.068 \mathrm{mg} / \mathrm{ml})$ compared to $L$. acidophilus $(0.137 \mathrm{mg} / \mathrm{ml})$, and C. albicans $(0.274 \mathrm{mg} / \mathrm{ml})$. The MBC values of the Cts-Se-NPs against the microorganisms after one, two, six, and 24 hours indicated that the concentration of $0.274 \mathrm{mg} / \mathrm{ml}$ of Cts-SeNPs completely killed S. mutans, L. acidophilus, and C. albicans after one, two, and six hours, respectively. At the concentration of $0.137 \mathrm{mg} / \mathrm{ml}, S$. mutans and L. acidophilus were killed after six and 24 hours, respectively. Conclusion: These findings encourage the potential use of Cts-Se-NPs in dentistry, while further clinical research is required in this area.
\end{abstract}

Keywords: Selenium; Chitosan; Streptococcus mutans; Lactobacillus acidophilus; Candida albicans. 


\section{Introduction}

Dental caries is one the most common disease problem in the world. The findings of a recent survey by the Global Oral Health Data Bank indicated the prevalence of dental caries to be 49-83\% [1]. Several types of microorganisms are associated with dental caries, but Streptococcus mutans and Lactobacillus acidophilus are the main bacteria involved in the initiation and progression of caries [2,3]. S. mutans, a gram-positive bacterium, is the most significant contributor to dental caries [4]. Lactobacillus species are the microbial markers of dental caries risk and a strong correlation has been reported between Lactobacillus counts and dental caries [5-7]. Lactobacillus acidophilus is the best-known species of gram-positive bacteria in the genus Lactobacillus [8].

Candida albicans is a commensal fungus that colonizes the human mucosal surfaces in the oral cavity [9] and is considered the most important Candida species, which cause oral infections. C. albicans is present in $30-60 \%$ of healthy individuals and $60-100 \%$ of denture wearers [10]. The coadhesion between S. mutans and C. albicans could lead to tooth surface colonization and enhance the microbial burden and production of the biofilm matrix, which is followed by severe tooth decay [11].

Numerous attempts have been made to develop optimal anticaries and antifungal agents. Recent findings have indicated that nanomaterials could be used as novel agents to prevent and treat dental caries [12]. In addition, several metal and metal oxide nanoparticles (NPs) have proven effective against cariogenic bacteria, such as silver, zinc oxide, magnesium oxide, titanium dioxides, gold, and copper oxide [13]. On the other hand, non-metallic NPs have been utilized as antibacterial and remineralizing agents; such examples are chitosan [14], hydroxyapatite [15], bioactive glass [16], and casein phosphopeptide-amorphous calcium phosphate [17-20]. Research development regarding nanomaterials' anticaries properties could result in the discovery of novel, effective agents against dental caries. The nano-size of selenium has attracted researchers' attention due to its excellent bioavailability and lower toxicity compared to the other forms of selenium [Q21]. The zero oxidation state of selenium (SeO) has low toxicity and excellent bioavailability, although it is unstable and should be stabilized by other biocompatible materials, such as chitosan [22]. Chitosan holds a special position among other biomaterials and is considered an ideal option for medical applications considering its biocompatibility, safety, biodegradability, and anti-inflammatory and antimicrobial activities [23].

Recently, we have investigated the antibacterial activity of colloidal selenium nanoparticles in chitosan solution (Cts-Se-NPs) as a new antibacterial agent [24], and the obtained results indicated that the Cts-Se-NP solution had excellent antibacterial activity against gram-positive bacteria (Streptococcus sanguinis, Staphylococcus aureus, and Enterococcus faecalis). In a continuation of our previous research, the present study aimed to investigate the antimicrobial activity of Cts-Se-NPs against S. mutans, L. acidophilus, and C. albicans. The novelty of the study lies on the antimicrobial and antifungal properties of Cts-Se-NPs against three cariogenic planktonic microorganisms.

\section{Material and Methods}

In this study, Cts-Se-NPs was synthesized according to our previous research [24]. Cts (0.15 g) was dissolved in a solution of $1.0 \%$ acetic acid solution at room temperature, 25 milliliters of ascorbic acid (0.01 $\mathrm{g} / \mathrm{ml}-1)$ was added to the Cts solution, and the solution was stirred. Afterwards, 25 milliliters of sodium selenite solution $(0.005 \mathrm{~g} / \mathrm{ml}-1)$ was added drop-wise to the solution and stirred to obtain a reddish-orange homogeneous colloid. Particle size measurements were performed using a particle size analyzer (SZ100, Horiba Ltd., Kyoto, Japan). 
The minimal inhibitory concentration (MIC) and minimal bactericidal concentration (MBC) were used as the most important predictive tools for the evaluation of antimicrobial activity [25]. MIC is defined as the lowest concentration of an antimicrobial agent that could inhibit the activity and growth of a microorganism after incubation. The broth microdilution method [26] in 96-well microplates was used to measure the MIC of the Cts-Se-NP solution against S. mutans, L. acidophilus, and C. albicans. Three oral microorganisms, S. mutans (PTCC No: 1683), L. acidophilus (PTCC No: 1643), and C. albicans (PTCC No: 5027), were obtained from Persian Type Culture Collection.

The twofold serial dilution was prepared using the Mueller Hinton broth (MHB). The twofold serial dilutions of the Cts-Se-NP solution in the MHB were transferred into the wells of a microplate and inoculated with 100 microliters of the bacterial suspension $\left(1-2 \times 10^{8} \mathrm{CFU} / \mathrm{ml}\right)$. McFarland Standard No. 0.5, used in this study, contains approximately cell density $\left(1-2 \times 10^{8} \mathrm{CFU} / \mathrm{ml}\right)$ and was determined by measuring the optical absorbance at a wavelength of $600 \mathrm{~nm}$. The absorbance was kept in the same range as equivalent to that of the McFarland standard 0.5 (Optical Density - O.D.) at $600 \mathrm{~nm}$ between 0.08-0.1 corresponds to $1-2 \times 10^{8}$ $\mathrm{CFU} / \mathrm{ml}$ ) [27]. The microplate was incubated at $37^{\circ} \mathrm{C}$ with $5 \% \mathrm{CO}_{2}$ in aerobic conditions for 24 hours, and the lowest concentration of the agent that prevented bacterial growth was recorded as the MIC. The bacteria inoculated into the MHB without Cts-Se NPs and culture media without the bacteria were considered as the positive and negative controls, respectively.

MBC is defined as the lowest concentration of an antibacterial agent killing most bacterial inoculums (99.99\%). To measure the MBC, 10 microliters of the bacterial suspensions in the wells were inoculated into the blood agar medium without turbidity and incubated at the temperature of $37^{\circ} \mathrm{C}$ until sufficient growth. The lowest concentration that killed 99.9\% (>3 $\log 10)$ of the initial inoculum after one, two, six, and 24 hours was considered as the MBC at each contact time. Figure 1 depicts the MIC and MBC tests of Cts-Se-NPs.

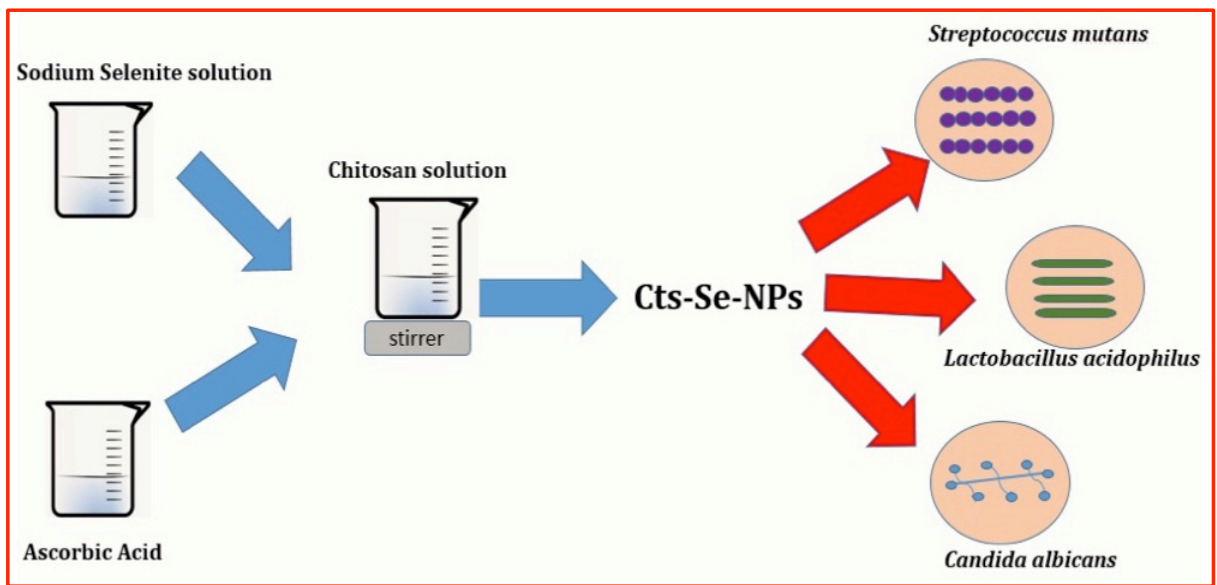

Figure 1. Scheme of synthesis of Cts-Se-NPs.

\section{Results}

As is observed in Figure 2, the mean particle size distribution of the Cts-Se NP solution was approximately 81.4 nanometers.

According to the obtained results, Cts-Se-NPs had significant antimicrobial activity against S. mutans, L. acidophilus, and C. albicans. The MIC values of Cts-Se-NPs were lowest for $S$. mutans $(68 \mu \mathrm{g} / \mathrm{ml})$ compared to L. acidophilus $(137 \mu \mathrm{g} / \mathrm{ml})$ and C. albicans $(274 \mu \mathrm{g} / \mathrm{ml})($ Table 1$)$. 


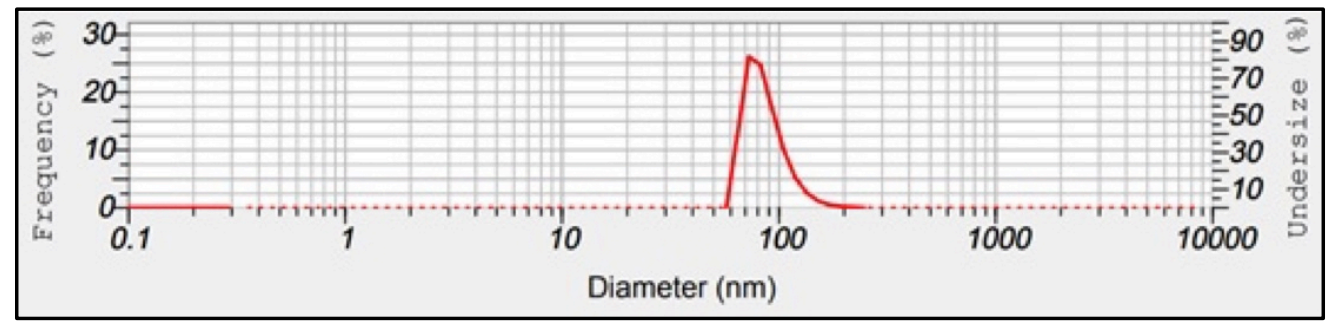

Figure 2. Particle Size Distribution of Cts-Se NPs.

Table 1. MIC values of Cts-Se-NPs.

\begin{tabular}{cc}
\hline Microorganisms & MIC \\
\hline Streptococcus mutans & $68 \mu \mathrm{g} / \mathrm{ml}$ \\
Lactobacillus acidophilus & $137 \mu \mathrm{g} / \mathrm{ml}$ \\
Candida albicans & $274 \mu \mathrm{g} / \mathrm{ml}$ \\
\hline
\end{tabular}

Table 2 shows the MBC values of Cts-Se-NPs against the microorganisms after one, two, six, and 24 hours. As can be seen, Cts-Se-NPs displayed more rapid bactericidal activity against $S$. mutans. Concentration of $274 \mu \mathrm{g} / \mathrm{ml}$ completely killed S. mutans, L. acidophilus, and C. albicans after one, two, and six hours, respectively. At the concentration of $137 \mu \mathrm{g} / \mathrm{ml}$, S. mutans and L. acidophilus were killed after six and 24 hours, respectively.

Table 2. MBC values of Cts-Se-NPs after one, two, six, and 24 hours.

\begin{tabular}{lcccc}
\hline \multicolumn{1}{c}{ Microorganisms } & \multicolumn{4}{c}{ MBC Values } \\
& After 1 Hour & After 2 Hours & After 6 Hours & After 24 Hours \\
\hline Streptococcus mutans & $274 \mu \mathrm{g} / \mathrm{ml}$ & $274 \mu \mathrm{g} / \mathrm{ml}$ & $137 \mu \mathrm{g} / \mathrm{ml}$ & $137 \mu \mathrm{g} / \mathrm{ml}$ \\
Lactobacillus acidophilus & $*$ & $274 \mu \mathrm{g} / \mathrm{ml}$ & $274 \mu \mathrm{g} / \mathrm{ml}$ & $137 \mu \mathrm{g} / \mathrm{ml}$ \\
Candida albicans & $*$ & $*$ & $274 \mu \mathrm{g} / \mathrm{ml}$ & $274 \mu \mathrm{g} / \mathrm{ml}$ \\
\hline *Not killed. & & &
\end{tabular}

\section{Discussion}

The present study aimed to investigate the antimicrobial activity of Cts-Se-NPs against three major microorganisms involved in dental caries, including S. mutans, L. acidophilus, and C. albicans.

The current research aimed to assess the antimicrobial activity of Cts-Se-NPs against S. mutans, L. acidophilus, and C. albicans. Previous research [24] confirmed the significant antibacterial effects of a Cts-Se-NP solution against three gram-positive bacteria (Streptococcus sanguinis, Staphylococcus aureus, and Enterococcus faecalis), while no such effects were observed on gram-negative bacteria.

According to the results of the present study, Cts-Se-NPs had remarkable antimicrobial effects against S. mutans, L. acidophilus, and C. albicans. In gram-positive bacteria, the cell wall is thick and consists of several layers of peptidoglycan without an outer lipopolysaccharide membrane. The lipopolysaccharides carry a net with a negative charge, causing the strong negative surface charge of these bacteria. The electrostatic interaction could easily deposit selenium NPs in the peptidoglycan layer of gram-positive bacteria, thereby disrupting the bacterial cell division [28]. The antibacterial activity of chitosan could be attributed to its cationic nature. Cationic materials are able to degrade the cell wall structure and cell membrane of bacteria, which in turn leads to the exposure of the cell membrane to osmotic shock and exudation of the cytoplasmic content, eventually followed by cell death [29]. In a study in this regard, Costa et al. [30] observed that 
chitosan was largely effective against the adherence and biofilm formation of $S$. mutans, and its action was evident in the inhibition of the initial adherence and biofilm formation and disruption of the mature biofilms. Similarly, Saita et al. [31] reported that submicron chitosan particles had significant antibacterial activity and anti-adhesive action against $S$. mutans.

In another study, Ikono et al. [32] investigated the effects of nanochitosan against S. mutans and C. albicans dual-species biofilms, reporting that $15 \%(\mathrm{v} / \mathrm{v})$ nanochitosan had prominent antimicrobial activity against the dual-species of $S$. mutans and C. albicans biofilms through decreasing the survival rate of the microbial cells. In addition, Costa et al. [30] compared the effects of a chitosan-based mouthwash with two commercial mouthwashes on the biofilm formation of S. mutans, L. acidophilus, E. faecium, C. albicans, and P. intermedia, reporting that the chitosan-based mouthwash exerted significant antibacterial effects against the microorganisms.

Several studies have also evaluated chitosan's antibacterial activity, although its mechanisms against C. albicans remain unclear [33]. For instance, Shih et al. [33] reported the mechanism of chitosan action against $C$. albicans through the inhibition of SAGA complex gene expression, which decreased the protection of the cell surface against chitosan. On the other hand, Krasniqi et al. [34] reported that selenium-based orthodontic bonding agent had significant effects against $S$. mutans, while Tran et al. [35] observed that selenium could inhibit bacterial plaque formation on human teeth with durable antibacterial properties.

In a study in this regard, Guisbiers et al. [36] stated that selenium NPs had remarkable antifungal activity against $C$. albicans and could easily adhere onto the biofilm, penetrate C. albicans, and disrupt the cell structure through substitution with sulfur. The effective adherence of selenium NPs onto C. albicans results from the higher adherence of C. albicans to materials with similar surface energy [36]. Due to the similar chemical properties of selenium and sulfur, they compete in biological processes, thereby leading to the substitution of sulfur by selenium in amino acids and the subsequent morphological changes in Candida cells; such examples are the increased cell size, shrinkage of yeasts, cytoplasm thickening, and changes in the vacuole structure [37].

Limitations are inevitable with in-vitro studies, such as the results may not correspond to the actual behaviors of Cts-Se-NPs in vivo because they are not exposed to the same conditions found in the oral cavity. In this study, only three cariogenic bacteria were investigated in laboratory, which might not show completely the antiplaque effect and substantivity property of Cts-Se-NPs. In vivo studies are required to support the efficacy of Cts-Se-NPs.

\section{Conclusion}

The use of Cts-Se-NPs is an effective approach to the inhibition of the three of the most common bacteria involved in dental caries. However, it is important to note that further studies are required to evaluate its effects on other aspects related to tooth caries.

\section{Authors' Contributions}

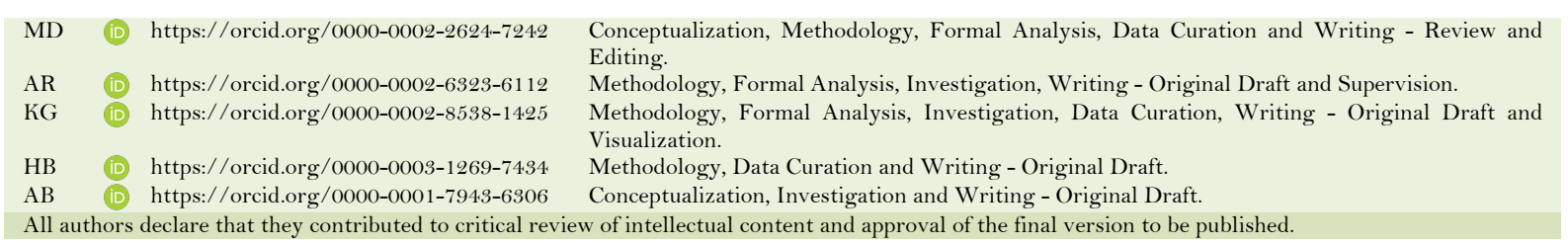




\section{Financial Support}

Mashhad University of Medical Sciences (Grant no. 961533).

\section{Conflict of Interest}

The authors declare no conflicts of interest.

\section{Data Availability}

The data used to support the findings of this study can be made available upon request to the corresponding author.

\section{References}

[1] Rathee M, Sapra A. Dental Caries. In: StatPearls. Treasure Island (FL): StatPearls Publishing; 2020.

[2] Elgamily H, Safy R, Makharita R. Influence of medicinal plant extracts on the growth of oral pathogens Streptococcus mutans and Lactobacillus acidophilus: an in-vitro study. Open Access Maced J Med Sci 2019; 7(14):2328-34. https://doi.org/10.3889/oamjms.2019.653

[3] Lemos JA, Palmer SR, Zeng L, Wen ZT, Kajfasz JK, Freires IA, et al. The biology of Streptococcus mutans. Microbiol Spectr 2019; 7(1):10.1128/microbiolspec.GPP3-0051-2018. https://doi.org/10.1128/microbiolspec.GPP3-0051-2018

[4] Cui T, Luo W, Xu L, Yang B, Zhao W, Cang H. Progress of antimicrobial discovery against the major cariogenic pathogen Streptococcus mutans. Curr Issues Mol Biol 2019; 32:601-44. https://doi.org/10.2 1775/cimb.032.601

[5] Granath L, Cleaton-Jones P, Fatti LP, Grossman ES. Salivary lactobacilli explain dental caries better than salivary mutans streptococci in 4-5-year-old children. Scand J Dent Res 1994; 102(6):319-23. https://doi.org/10.1111/j.1600-0722.1994.tbo1477.x.-

[6] Zickert I, Emilson CG, Krasse B. Streptococcus mutans, lactobacilli and dental health in 13-14-year-old Swedish children. Community Dent Oral Epidemiol 1982; 1O(2):77-81. https://doi.org/10.1111/j.1600-0528.1982.tbo0367.x

[7] Köhler B, Bjarnason S. Mutans streptococci, lactobacilli and caries prevalence in 11-and 12-year-old Icelandic children. Community Dent Oral Epidemiol 1987; 15(6):332-5. https://doi.org/10.1111/j.1600-0528.1987.tb01747.x

[8] Ozogul F, Hamed I. Lactic acid bacteria: Lactobacillus spp.: Lactobacillus acidophilus. Reference Module in Food Science 2016. https://doi.org/10.1016/b978-0-08-100596-5.00852-0

[9] Kumar R, Breindel C, Saraswat D, Cullen PJ, Edgerton M. Candida albicans Sap6 amyloid regions function in cellular aggregation and zinc binding, and contribute to zinc acquisition. Sci Rep 2017; 7(1):1-15. https://doi.org/10.1038/s41598-017-03082-4

[10] Gacon I, Loster JE, Wieczorek A. Relationship between oral hygiene and fungal growth in patients: users of an acrylic denture without signs of inflammatory process. Clin Interv Aging 2019; 14:1297-1302. https://doi.org/10.2147/CIA.S193685

[11] Koo H, Andes DR, Krysan DJ. Candida-streptococcal interactions in biofilm-associated oral diseases. PLoS Pathog 2018; 14(12):e1007342. https://doi.org/10.1371/journal.ppat.1007342

[12] Noori AJ, Kareem FA. The effect of magnesium oxide nanoparticles on the antibacterial and antibiofilm properties of glass-ionomer cement. Heliyon 2019; 5(10):e02568. https://doi.org/10.1016/j.heliyon.2019.e02568

[13] Carrouel F, Viennot S, Ottolenghi L, Gaillard C, Bourgeois D. Nanoparticles as anti-microbial, anti-inflammatory, and remineralizing agents in oral care cosmetics: a review of the current situation. Nanomaterials 2020; $10(1): 140$. https://doi.org/10.3390/nano10010140

[14] Husain S, Al-Samadani KH, Najeeb S, Zafar MS, Khurshid Z, Zohaib S, et al. Chitosan biomaterials for current and potential dental applications. Materials 2017; 10(6):602. https://doi.org/10.3390/ma 10060602

[15] Pepla E, Besharat LK, Palaia G, Tenore G, Migliau G. Nano-hydroxyapatite and its applications in preventive, restorative and regenerative dentistry: a review of literature. Ann Stomatol 2014; 5(3):108-14.

[16] Skallevold HE, Rokaya D, Khurshid Z, Zafar MS. Bioactive glass applications in dentistry. Int J Mol Sci 2019; 2O(23):5960. https://doi.org/10.3390/ijms20235960

[17] Ma X, Lin X, Zhong T, Xie F. Evaluation of the efficacy of casein phosphopeptide-amorphous calcium phosphate on remineralization of white spot lesions in vitro and clinical research: a systematic review and meta-analysis. BMC Oral Health 2019; 19(1):295. https://doi.org/10.1186/s12903-019-0977-0

[18] Heravi F, Bagheri H, Rangrazi A, Zebarjad SM. Effects of the addition of casein phosphopeptide-amorphous calcium phosphate (CPP-ACP) on mechanical properties of luting and lining glass ionomer cement. Mater Res Express 2016; 3(7):075405. https://doi.org/10.1088/2053-1591/3/7/075405

[19] Heravi F, Bagheri H, Rangrazi A, Zebarjad SM. Incorporation of CPP-ACP into luting and lining GIC: Influence on wear rate (in the presence of artificial saliva) and compressive strength. ACS Biomater Sci Eng 2016; 2(11):1867-71. https://doi.org/10.1021/acsbiomaterials.6boo204 
[20] Heravi F, Bagheri H, Rangrazi A, Zebarjad SM. An in vitro study on the retentive strength of orthodontic bands cemented with CPP-ACP-containing GIC. Mater Res Express 2016; 3(12):125401.

https://doi.org/10.1088/2053-1591/3/12/125401

[21] Vahdati M, Moghadam TT. Synthesis and characterization of selenium nanoparticles-lysozyme nanohybrid system with synergistic antibacterial properties. Sci Rep 2020; 10(1):510. https://doi.org/10.1038/s41598-019-57333-7

[22] Zhai X, Zhang C, Zhao G, Stoll S, Ren F, Leng X. Antioxidant capacities of the selenium nanoparticles stabilized by chitosan. J Nanobiotechnology 2017; 15(1):4. https://doi.org/10.1186/s12951-016-0243-4

[23] Islam MM, Shahruzzaman M, Biswas S, Sakib MN, Rashid TU. Chitosan based bioactive materials in tissue engineering applications - $\mathrm{A}$ review. Bioact Mater 2020; 5(1):164-83. https://doi.org/10.1016/j.bioactmat.2020.01.012

[24] Rangrazi A, Bagheri H, Ghazvini K, Borouziniat A, Darroudi M. Synthesis and antibacterial activity of colloidal selenium nanoparticles in chitosan solution: a new antibacterial agent. Mater Res Express 2020; 6(12):1250h3. https://doi.org/10.1088/2053-1591/ab6a56

[25] Brown D, Blowers R. Disc Methods of Sensitivity Testing and Other Semiquantitative Methods. In: Reeves DS, Phillips I, Williams JD, Wise R. Laboratory Methods in Antimicrobial Chemotherapy. Edinburgh: Livingstone; 1978.

[26] Qaiyumi S. Macro-and Microdilution Methods of Antimicrobial Susceptibility Testing. Antimicrobial Susceptibility Testing Protocols. Boca Raton: Taylor \& Francis; 2007. p. 75-79.

[27] McFarland J. The nephelometer: an instrument for estimating the number of bacteria in suspensions used for calculating the opsonic index and for vaccines. JAMA 1907; 49(14):1176-8. https://doi.org/10.1001/jama.1907.25320140022001f

[28] Tran PA, O’Brien-Simpson N, Reynolds EC, Pantarat N, Biswas DP, O’Connor AJ. Low cytotoxic trace element selenium nanoparticles and their differential antimicrobial properties against $\mathrm{S}$. aureus and E. coli. Nanotechnology 2015; 27(4):045101. https://doi.org/10.1088/0957-4484/27/4/045101

[29] Rajabnia R, Ghasempour M, Gharekhani S, Gholamhoseinnia S, Soroorhomayoon S. Anti-Streptococcus mutans property of a chitosan: Containing resin sealant. J Int Soc Prev Community Dent 2016; 6(1):49-53. https://doi.org/10.4103/2231-0762.175405

[30] Costa E, Silva S, Tavaria F, Pintado M. Study of the effects of chitosan upon Streptococcus mutans adherence and biofilm formation. Anaerobe 2013; 20:27-31. https://doi.org/10.1016/j.anaerobe.2013.02.002

[31] Saita K, Nagaoka S, Shirosaki T, Horikawa M, Ihara H. Dispersible chitosan particles showing bacteriostatic effect against Streptococcus mutans and their dental polishing effect. Biosci Biotechnol Biochem 2020; 84(6):1265-73. https://doi.org/10.1080/09168451.2020.1723402

[32] Ikono R, Vibriani A, Wibowo I, Saputro KE, Muliawan W, Bachtiar BM, et al. Nanochitosan antimicrobial activity against Streptococcus mutans and Candida albicans dual-species biofilms. BMC Res Notes 2019; 12(1):383. https://doi.org/10.1186/s13104-019-4422-x

[33] Shih P-Y, Liao Y-T, Tseng Y-K, Deng F-S, Lin C-H. A potential antifungal effect of chitosan against Candida albicans is mediated via the inhibition of SAGA complex component expression and the subsequent alteration of cell surface integrity. Front Microbiol 2019; 10:602. https://doi.org/10.3389/fmicb.2019.00602

[34] Krasniqi S, Sejdini M, Stubljar D, Jukic T, Ihan A, Aliu K, et al. Antimicrobial effect of orthodontic materials on cariogenic bacteria Streptococcus mutans and Lactobacillus acidophilus. Med Sci Monit Basic Res 2020; $26:$ e920510. https://doi.org/10.12659/MSMBR.920510

[35] Tran P, Hamood A, Mosley T, Gray T, Jarvis C, Webster D, et al. Organo-selenium-containing dental sealant inhibits bacterial biofilm. J Dent Res 2013; 92(5):461-6. https://doi.org/0.1177/0022034513482141

[36] Guisbiers G, Lara HH, Mendoza-Cruz R, Naranjo G, Vincent BA, Peralta XG, et al. Inhibition of Candida albicans biofilm by pure selenium nanoparticles synthesized by pulsed laser ablation in liquids. Nanomedicine 2017; 13(3):1095-103. https://doi.org/10.1016/j.nano.2016.10.011

[37] Kieliszek M, Błażejak S, Bzducha-Wróbel A, Kurcz A. Effects of selenium on morphological changes in Candida utilis ATCC 9950 yeast cells. Biol Trace Elem Res 2016; 169(2):387-93. https://doi.org/10.1007/s12011-015-0415-3 